\title{
Review of: "Multi-endpoint analysis of human 3D airway epithelium following repeated exposure to whole electronic vapor product aerosol or cigarette smoke"
}

Hongguang $\mathrm{Nie}^{1}$

1 China Medical University Shenyang

Potential competing interests: The author(s) declared that no potential competing interests exist.

In this manuscript, Czekala L. et al. studied the effects of repeated whole cigarette smoke and whole EVP aerosol exposure to a 3D lung model at the ALI. The setup of 3D lung models for the assessments of human-relevant product risk is promising. The following concerns might be considered by the authors,

1. '3D lung model' is actually isolated from upper airway human epithelium, therefore '3D human airway tissue model' might be better.

2. Figure $3 \mathrm{~B}$, too much blank space exists.

3. The relationship of barrier integrity and inflammation should be discussed in more detail.

4. How to explain the difference of TEER values in 90 puffs of 3R4F smoke and myblu aerosol? 